\title{
Dissecting Peroxisome-Mediated Signaling Pathways: a New and Exciting Research Field
}

\author{
Bo Wang, Oksana Apanasets, Marcus Nordgren, and Marc \\ Fransen
}

Laboratory of Lipid Biochemistry and Protein Interactions, Department of Cellular and

Molecular Medicine, KU Leuven, Belgium

Corresponding author: Marc Fransen (marc.fransen@med.kuleuven.be)

\begin{abstract}
Peroxisomes are multifunctional organelles that play an important role in the metabolism of lipids and reactive oxygen species. As many cellular signaling functions are regulated via lipids, lipid second messengers, and oxidative stress-related factors, it is not surprising to see that these organelles are increasingly recognized as critical regulators of cellular signaling events. To fulfill these signaling functions, peroxisomes physically and functionally interact with other cell organelles, including mitochondria. Recent progress in the development of tools to visualize and modulate molecular processes at the subcellular level has made it possible to gain a better insight into the potential mechanisms governing peroxisomal signaling. This chapter is intended to provide a comprehensive overview of the tools and strategies that are currently available to study peroxisomemediated signaling pathways in living cells. To provide the reader with relevant background information, we also highlight key studies that have contributed to our understanding of how peroxisomes may function as important sites of redox-, lipid-, inflammatory-, and viral-mediated signal transduction.
\end{abstract}

\section{Introduction}

Peroxisomes are functionally complex organelles that play a central role in diverse metabolic processes. These processes can vary widely between species, cell types, and physiological and developmental 
stages (Fransen 2012). For a long time, peroxisomes were thought to function solely as metabolic organelles. However, over the last years, it has become increasingly clear that peroxisome-derived metabolites can exert cytoprotective effects by activating pro-survival pathways or initiate signaling cascades that ultimately induce pathophysiological responses (Titorenko and Terlecky 2011). In addition, peroxisomes are increasingly recognized as signaling platforms in the battle between viruses and their hosts (Dixit et al. 2010; Lazarow 2011). In the following sections, we first review the emerging concepts highlighting the potential role of peroxisomes in diverse signaling pathways, with an emphasis on mammals. Afterwards, we provide an overview of tools and approaches that are currently available to explore peroxisome-mediated signaling events.

\section{Peroxisomes as Signaling Platforms}

It is currently a common belief that most, if not all, intracellular signaling pathways are controlled by redox regulation and/or lipid second messengers. In addition, it is well known that the specific cellular responses to signaling molecules depend on their identity, concentration, and spatial distribution. Given that peroxisomes actively contribute to the bioavailability of various reactive oxygen and nitrogen species (ROS/RNS), bioactive lipids, and inflammatory factors (see below), it is not surprising to see that these organelles are increasingly recognized as important intracellular signaling platforms (Fig. 1) (Beach et al. 2012). The following sections are intended to summarize key findings in this rapidly moving and exciting research area. Importantly, the exploration of this research field has just begun, and as such, there is still a large gap in our understanding of how peroxisomes are incorporated into subcellular communication networks.

\subsection{Redox Signaling}

For a long time, it was thought that ROS/RNS were only toxic byproducts of aerobic metabolism. However, in the meantime, it has 
become clear that some ROS/RNS (e.g. hydrogen peroxide $\left(\mathrm{H}_{2} \mathrm{O}_{2}\right)$ and nitric oxide $\left(\mathrm{NO}^{\circ}\right)$ ) can act as intracellular messengers at subtoxic concentrations (Fransen et al. 2012). In general, this signaling occurs through the oxidative modification of reactive cysteine residues. In this context, it should be mentioned that many signaling components like kinases, phosphatases, and transcription factors contain cysteine residues that can be reversibly modified in a redoxresponsive manner (Barford 2004). Examples of such modifications include, but are not limited to, disulfide formation, S-nitrosylation, and S-glutathionylation. As these modifications often influence protein activity, a chronic or acute disturbance in redox homeostasis can be expected to deregulate vital cellular signaling pathways. This may influence cell fate by, for example, promoting cell growth or death.

Almost 60 years after their discovery, there is a wealth of evidence supporting the idea that peroxisomes can function as important redox signaling nodes (Fig. 1) (Del Rio 2011). In the following paragraphs, we will elaborate on what is known about this topic in mammals. For a detailed overview of the small reactive molecules that can be produced and degraded within the peroxisomal matrix, the enzymes that are involved in these processes, and the potential mechanisms by which ROS/RNS are translocated across the peroxisomal membrane, we refer the reader to other recent reviews (Antonenkov et al. 2010; Fransen et al. 2012).

As can be inferred from their name, peroxisomes play a central role in the cellular metabolism of $\mathrm{H}_{2} \mathrm{O}_{2}$. This is perhaps best illustrated by the observation that about $35 \%$ of all $\mathrm{H}_{2} \mathrm{O}_{2}$ formed in rat liver is derived from peroxisomal oxidases (Boveris et al. 1972). Strong support that peroxisomal $\mathrm{H}_{2} \mathrm{O}_{2}$ fluxes can effectively influence cellular signaling events comes from multiple in cellulo and in vivo studies. For example, overexpression of acyl-CoA oxidase 1 (ACOX1), a $\mathrm{H}_{2} \mathrm{O}_{2}$-producing peroxisomal enzyme, can activate the redox-sensitive transcription factor nuclear factor $\kappa \mathrm{B}(\mathrm{NF}-\kappa \mathrm{B})$ in a substrate concentration-dependent manner ( $\mathrm{Li}$ et al. 2000); inhibiting the activity of catalase, a $\mathrm{H}_{2} \mathrm{O}_{2}$-decomposing peroxisomal enzyme, can increase the cellular protein disulfide content by $20 \%$ (Yang et al. 2007); overexpression of catalase can sensitize cells to paraquat- 
and TNF $\alpha$-induced cell death by dampening $\mathrm{H}_{2} \mathrm{O}_{2}$-mediated signaling pathways (Chen et al. 2004); and endogenous catalase plays an important role in protecting the kidney from diabetic stress through maintaining peroxisomal and mitochondrial fitness (Hwang et al. 2012).

Mammalian peroxisomes are also involved in the production and degradation of other ROS/RNS species, such as superoxide $\left(\mathrm{O}_{2}{ }^{-}\right)$, $\mathrm{NO}^{\circ}$, and peroxynitrite (ONOO${ }^{-}$) (Fransen et al. 2012). Unfortunately, with few exceptions, virtually nothing is known regarding the specific effects and physiological functions of these peroxisomal ROS/RNS species. However, it should be pointed out that there are some indications that the peroxisomal pool of NOS2, the inducible nitric oxide synthase, may function as a local enzyme activitymodulating factor (Stolz et al. 2002). This hypothesis is in agreement with the findings that the appearance of NOS2 inside peroxisomes has been associated with a decrease in catalase activity (Stolz et al. 2002), and various peroxisomal proteins, including catalase, can be S-nitrosylated (Doulias et al. 2013).

Other strong but indirect evidence that peroxisomes can indeed function as redox signaling platforms comes from studies in mice. For example, it has been shown that (i) preservation of peroxisome function is essential to reduce renal ROS levels and alleviate kidney injury after ischemia/reperfusion or cisplatin treatment (Hasegawa et al. 2010), (ii) peroxisomal ROS metabolism plays a key role in the regulation of the hypothalamic melanocortin tone and food intake in diet-induced obesity (Diano et al. 2011), and (iii) the accumulation of functionally compromised peroxisomes alters the cellular redox equilibrium and attenuates organ injury induced by lipopolysaccharides (Vasko et al 2013). Again, the molecular mechanisms of how peroxisomes exactly contribute to these physiological and pathological redox signaling processes are poorly understood. However, here it is essential to mention that peroxisomes share an intricate redoxsensitive relationship with mitochondria (Ivashchenko et al. 2011; Walton and Pizzitelli 2012). This in turn suggests that mitochondria may act as dynamic receivers, integrators, and transmitters of peroxisome-derived mediators of oxidative stress. For more information regarding how peroxisomes and mitochondria can communicate 
with each other, we refer the reader to another recent review (Fransen et al. 2013).

\subsection{Lipid and Inflammatory Signaling}

Many cellular signaling functions are regulated via lipids and lipid second messengers. Remarkably, despite the fact that peroxisomes are actively involved in the metabolism of many of these compounds, very little is known about how these organelles contribute to cross-compartmental lipid signaling. However, as (i) peroxisomes harbor enzymes that are involved in the biosynthesis of plasmalogens and docosahexaenoic acid (Van Veldhoven 2010), (ii) these lipids function as potential reservoirs for bioactive molecules such as prostaglandins, thromboxanes, leukotrienes, resolvins, docosatrienes, and neuroprotectins (Braverman and Moser 2012), and (iii) some of these molecules (e.g. the eicosanoids) can also be degraded through peroxisomal $\beta$-oxidation (Van Veldhoven 2010), it comes as no surprise that these organelles are garnering increasing attention as signaling platforms in inflammation and immunoregulation (Fig. 1). This is evidenced by the following observations: peroxisomes provide oligodendrocytes with an essential protective function against axon degeneration and neuroinflammation (Kassmann et al. 2007); abnormal accumulation of very-long-chain fatty acids, a condition associated with peroxisome dysfunction, can lead to an increase in inflammatory cytokine expression (Singh et al. 2009); the interleukin-1 signaling pathway is significantly activated in skin fibroblasts from patients lacking functional ACOX1 (El Hajj et al. 2012); and inactivation of neuronal multifunctional protein 2 , a central enzyme of peroxisomal $\beta$-oxidation, causes neuroinflammation (Verheijden et al. 2013).

Over the years, it has become increasingly clear that lipid signaling, inflammation and oxidative stress are inextricably linked processes. In addition, it is well known that virtually all stress stimuli trigger changes in lipid composition. For example, exposure of cells to oxidative stress can induce sphingolipid metabolism and lipid peroxidation (Bikman and Summers, 2011). This in turn may lead to the ac- 
cumulation of ceramides, ceramide metabolites, and highly reactive electrophilic aldehydes. Importantly, as (i) many of these compounds can act as important messengers in signaling events that lead to cell proliferation, differentiation, and senescence (Chen and Niki 2006; Bikman and Summers 2011), and (ii) brains and fibroblasts of patients and mice with peroxisomal disorders display a significant increase in the level of C26:1/0-ceramide (Pettus et al. 2004) and lipid peroxidation products (Fourcade et al. 2008; Baarine et al. 2012), it is very likely that changes in peroxisomal metabolism can directly or indirectly modulate cytoprotective and cytotoxic signal transduction pathways (Titorenko and Terlecky 2011). This idea is further corroborated by the observation that cells lacking GNPAT, a peroxisomal enzyme catalyzing the first step in ether phospholipid biosynthesis, are more vulnerable to oxidative stress (Brodde et al. 2012). Note that plasmalogens, among other functions, have been found to possess antioxidant capacities (Braverman and Moser 2012).

\subsection{Innate Immune Signaling}

Innate immunity provides the first line of defense against pathogen invasion. The initiation of innate immune responses relies on the recognition of pathogen components by pattern recognition receptors (PRRs). Activated PRRs deliver signals to specific adaptor proteins that, in turn, orchestrate complex host defense mechanisms. These include the activation of transcription factors (e.g. NF- $\kappa \mathrm{B}$, activator protein 1, and interferon regulatory factors (IRFs)) and the subsequent production of type 1 interferons (IFNs), inflammatory cytokines, and chemokines (Chen and Jiang 2013). Interestingly, a few years ago, it was reported that a small portion of mitochondrial antiviral signaling protein (MAVS), a RIG-I-like receptor (RLR) adaptor protein, is located on peroxisomes (Fig. 1) (Dixit et al. 2010).

RLRs are a family of cytosolic PRRs that function as innate immune receptors for specific RNA virus ligands. Kagan and coworkers also found that (i) RNA viruses can activate MAVS-dependent signaling from peroxisomes, (ii) this signaling process is temporally and qualitatively different from that of mitochondrial MAVS, and (iii) MAVS 
responses from both peroxisomes and mitochondria are needed for maximal antiviral activity (Dixit et al. 2010). Recently, these findings have been extended by the observation that also LSm14A, a processing body-associated sensor of viral RNA and DNA, is translocated to peroxisomes upon viral infection ( $\mathrm{Li}$ et al. 2012). Importantly, this process is also MAVS-dependent and mediates IRF3 activation and IFN- $\beta$ production ( $\mathrm{Li}$ et al. 2012).

Over the last years, evidence has accumulated suggesting that some viruses exploit peroxisomes for their replication. Unfortunately, the precise role of these organelles in viral replication is yet to be determined. For a state-of-the-art overview of research in this field, we refer the reader to another review (Lazarow 2011). Finally, there are indications that peroxisomes may also modulate inflammatory immune responses. For example, it has been shown that a peroxisome deficiency in Drosophila larvae causes an increase in the expression of genes involved in innate immunity and humoral responses (Mast et al. 2011), and that the immune system is activated in nestin-Pex5 knockout mice (Bottelbergs et al. 2012). Again, the molecular mechanisms underlying these phenomena remain to be fully elucidated. However, it has been hypothesized that the upregulation of innate immunity genes may be a response to increased levels of unused peroxisomal metabolites (Mast et al. 2011).

\section{Tools to Study Peroxisome-Mediated Signaling Pathways}

Signaling pathways can be considered as cascades of biochemical reaction-diffusion processes that govern cellular functions in a spatiotemporal manner. Many of these processes are triggered by transient changes in the internal or external milieu (e.g. nutrient status, $\mathrm{Ca}^{2+}$ levels, $\mathrm{pH}$, redox state, etc.) and ultimately lead to transcriptional reprogramming. As a dysregulation of these signaling pathways often contributes to the etiology and progression of disease, it is critical to understand these processes at the molecular and (sub)cellular level. During recent years, the development of new methods to visualize proteins and biochemical reactions in function of time and space in living cells has revolutionized signaling re- 
search. In the next sections, we further elaborate on the tools and strategies that are currently available to study peroxisome-mediated signaling pathways in living cells (Fig. 1). We first briefly outline different strategies that can be used to visualize (distinct populations of) peroxisomes by direct fluorescence microscopy (see Sect. 3.1). Next, we discuss a number of tools that can be used to detect and modulate peroxisomal signaling activity in a spatially and temporally controlled manner (see Sect. 3.2). Note that, as (i) the continuous development of new and improved synthetic and genetically encoded probes offers an increasing array of possibilities for imaging spatiotemporal processes in living cells, and (ii) it is impossible to outline all these possibilities and variations, we limit ourselves to an overview of probes that can be used to track peroxisomal redox changes and ion fluctuations.

\subsection{Fluorescence Microscopic Methods to Visualize Peroxisomes in Living Cells}

About half a century ago, peroxisomes were first visualized in rat liver by electron microscopy (Rhodin 1954). This technique, which is still widely used today, was for a long time the most prominent if not only - approach to get a visual image of these organelles.

However, as (i) the use of electron microscopy to image biological systems has some intrinsic limitations (e.g. no live samples, high cost and time expenses, etc.), and (ii) during the last decade, rapid progress has been made in the area of live-cell imaging by fluorescence microscopy (e.g. development of sensitive and specific fluorescent probes for functional analysis), the latter technology has often become the first method of choice to study the subcellular localization and dynamics of ions, metabolites, signaling molecules, and proteins (Wang et al. 2012). In the next paragraph, we highlight how some of these advances have been applied to study peroxisome dynamics. Here it is important to note that peroxisomes can rapidly adapt their morphology and number in response to changes in the cellular environment (Ribeiro et al. 2012). 
Most strategies that are currently employed to visualize peroxisomes in living cells make use of fluorescent proteins (FPs) that are fused to peroxisomal signal peptides (e.g. PTS1, PTS2, or mPTS) via recombinant DNA techniques. Although these probes are excellent tools for monitoring the import capacity, appearance, and trafficking of the total population of peroxisomes, they do not allow researchers to optically distinguish pools of proteins and organelles that are synthesized at different time points. To deal with this problem, two strategies have already been adopted from other research fields. The first one utilized a photoactivatable green FP (PAGFP) to investigate peroxisome maturation from the ER (Kim et al. 2006). Photoactivatable FPs are capable to change their spectral properties in response to irradiation with light of specific wavelengths, thereby enabling the spatial and temporal visualization of specific structures and tracking of their signal in time. A potential disadvantage of many of these proteins is that optimal photoactivation requires intense irradiation with UV light, a condition known to cause oxidative stress. The second strategy exploited the HaloTag technology to study peroxisome growth, division, and degradation (Huybrechts et al. 2009; Delille et al. 2010). This technology is a chemistry-based method for protein labeling in which synthetic chloroalkane ligands covalently bind to a dehalogenase-based protein fusion tag. As (i) the chloroalkane linker can be attached to different membrane-permeable functional groups (e.g. fluorophores, affinity handles, etc.), (ii) these probes spontaneously react with the HaloTag fusion protein, (iii) unbound probes can be easily washed away to reduce the signal-tonoise ratio, and (iv) labeling of newly-synthesized proteins can be completely turned off by using the HaloTag blocking ligand, this strategy is very well suited to conduct pulse-chase experiments in cells expressing a HaloTag-fusion protein (Los et al. 2008). This technology is also perfectly suited to visualize distinct pools of proteins, generated from a single genetic HaloTag-encoding construct, by sequentially incubating the cells with different fluorescent ligands. In the context of this chapter, it is worth mentioning that the HaloTag technology has been used to show that a disturbance in peroxisomal redox balance may function as a trigger for peroxisome degradation (Ivashchenko et al. 2011). As for PAGFP, a potential drawback of the HaloTag technology is the relative large size $( \pm 33$ $\mathrm{kDa}$ ) of the protein tag (Los et al. 2008). However, alternative 
strategies employing fluorogenic probes in combination with small protein tags (e.g. the $1 \mathrm{kDa}$ tetracysteine tag) are currently available and have already been applied successfully in related research fields (Hori and Kikuchi 2013). Another attractive approach that can potentially be adopted to simultaneously track the localization and age of individual peroxisomes is the use of fluorescent timers, which change their color with time due to a chemical conversion of the chromophore (Chudakov et al. 2010).

\subsection{Indicators and Modulators of Peroxisomal Signal Transduction}

\subsubsection{Redox Signaling}

Peroxisomes are actively involved in cellular ROS metabolism, and alterations in the peroxisomal redox state are likely to have important consequences on cellular physiology (see Sect. 2). As such, it is essential to gain a better understanding of how changes in peroxisomal redox metabolism impact cell signaling pathways. A requirement to carry out such studies is to have access to tools that are suitable to detect and modulate the peroxisomal redox state. In the next two sections, we discuss approaches that are currently used for these purposes.

\section{Redox Sensors}

$\mathrm{H}_{2} \mathrm{DCFDA}$ - The cell-permeable indicator dye 2', 7'-

dichlorodihydrofluorescein diacetate $\left(\mathrm{H}_{2} \mathrm{DCFDA}\right)$ is one of the most popular probes to assess oxidative stress in living cells. After passing through the membrane, this non-fluorescent lipophilic compound is de-esterified to a hydrophilic alcohol $\left(\mathrm{H}_{2} \mathrm{DCF}\right)$ that can be oxidized to 2', 7'-dichlorofluorescein (DCF), a highly fluorescent molecule $\left(\mathrm{E}_{\mathrm{ex}}=502 \mathrm{~nm} ; \mathrm{E}_{\mathrm{em}}=523 \mathrm{~nm}\right)($ Karlsson et al. 2010).

$\mathrm{H}_{2}$ DCFDA, which is thought to function as a general oxidative stress indicator, is also commonly used in the peroxisome field. For exam- 
ple, this compound has been employed to demonstrate that ROS levels are markedly increased in fibroblasts from X-linked adrenoleukodystrophy patients (Fourcade et al. 2008). Importantly, the use of $\mathrm{H}_{2}$ DCFDA to evaluate cellular oxidative stress faces several limitations: oxidation of $\mathrm{H}_{2} \mathrm{DCF}$ to DCF is an irreversible process; DCF fluorescence may not always be a result of exposure to ROS, but may also reflect the relocation of lysosomal iron and/or mitochondrial cytochrome $\mathrm{c}$ to the cytosol; DCF itself can also generate ROS upon visible light illumination; and the compound can not be targeted to specific subcellular compartments (Karlsson et al 2010).

Dihydroethidine - Another compound that can be used to visualize ROS production in living cells is dihydroethidine (DHE). DHE is a cell-permeable non-targetable redox sensor that reacts preferentially with $\mathrm{O}_{2}{ }^{--}$to form 2-hydroxyethidium (Dikalov et al. 2007). This molecule rapidly intercalates into double-stranded DNA, and this in turn results in a marked increase in nuclear fluorescence $\left(\mathrm{E}_{\mathrm{ex}}=510\right.$ $\mathrm{nm} ; \mathrm{E}_{\mathrm{em}}=590 \mathrm{~nm}$ ). DHE has already been successfully used to demonstrate that $\mathrm{O}_{2}{ }^{--}$production in peroxisome-deficient cerebellar neurons was almost twice that of littermate controls (Müller et al. 2011). Note that, as (i) DHE is light-sensitive, (ii) in cells and tissues, DHE can be oxidized to ethidium in a $\mathrm{O}_{2}{ }^{--}$-independent manner, and (iii) ethidium is difficult to distinguish from 2hydroxyethidium by conventional fluorescence techniques upon excitation at $510 \mathrm{~nm}$, care should be taken when interpreting results (Dikalov et al. 2007).

Redox-sensitive green fluorescent proteins - Approximately a decade ago, Remington and colleagues developed novel GFP probes suitable for monitoring redox changes in individual cells (Hanson et al. 2004). These probes, called roGFPs, contain two engineered cysteine residues on adjacent surface-exposed $\beta$-strands close to the chromophore. Disulfide formation between these residues promotes protonation of the chromophore, and this in turn increases the protein's excitation peak near $400 \mathrm{~nm}$ at the expense of the peak near $480 \mathrm{~nm}$. As oxidation of the thiol pair causes reciprocal changes in the emission intensities (around $510 \mathrm{~nm}$ ) when excited at these two different wavelengths, the fluorescence ratios can provide accurate insight into the redox environment of the chromophore. Importantly, 
the use of genetically encoded ratiometric fluorescent probes alleviates several shortcomings of chemical redox sensors. For example, the results will not depend on sensor concentration, cellular thickness, and the sensor distribution pattern; these probes show reversible changes in fluorescence; and they can be targeted to specific subcellular locations (Schwartzländer et al. 2008). Importantly, a variety of roGFP biosensors has been developed and characterized (Lukyanov and Belousov 2013). These include, among others, roGFP2, Grx1-roGFP2 and Orp1-roGFP2. RoGFP2 preferentially interacts with glutaredoxins (Grxs) and is therefore particularly suited to monitor changes in the glutathione redox couple (GSH/GSSG) (Hanson et al. 2004). However, as the availability of Grxs is often a rate-limiting factor in roGFP2-equilibration with intracellular thiols, the response of this probe to changes in redox potential is rather slow. To solve this problem, Dick and colleagues fused roGFP2 to human Grx1 and demonstrated that the oxidation of this fusion protein by GSSG is at least 100,000 times faster as compared to uncoupled roGFP2 (Gutscher et al. 2009). Next, the same research group demonstrated that fusing roGFP2 to the yeast peroxidase Orp1 resulted in a $\mathrm{H}_{2} \mathrm{O}_{2}$-specific probe with the spectral properties of roGFP2. In the meantime, we and others have shown that a peroxisome-targeted variant of roGFP2 is sufficiently sensitive to detect changes in the peroxisomal redox environment in response to altered growth conditions or upon challenge with cell-permeant oxidants and reductants, toxicologically relevant metal ions, or green light illumination after peroxisomal KillerRed expression (Schwartzländer et al. 2008; Ivashchenko et al. 2011).

Redoxfluor - Redoxfluor is a fluorescence resonance energy transfer (FRET)-based redox sensor, which consists of three parts: cerulean, a variant of enhanced cyan fluorescent protein (CFP); a tandemly repeated fragment of a partial region within the carboxy-terminal cysteine-rich domain of Yap1, a yeast transcription factor which activates expression of antioxidant genes in response to oxidative stress; and citrine, a variant of enhanced yellow fluorescent protein (YFP) (Yano et al. 2010). Upon oxidation and excitation at $434 \mathrm{~nm}$, CFP emission (near $476 \mathrm{~nm}$ ) is increased at the expense of YFP emission (near $527 \mathrm{~nm}$ ), thereby decreasing the yellow-to-cyan emission ratio. Importantly, Redoxfluor does not measure a specific 
type of ROS. It rather detects quantitative changes of various redoxrelated compounds such as $\mathrm{H}_{2} \mathrm{O}_{2}$, glutathione, and thioredoxin. Redoxfluor has already been successfully used to compare the redox state within peroxisomes and the cytosol in yeast and Chinese hamster ovary cells harboring functional or dysfunctional peroxisomes (Yano et al. 2010). One interesting observation of this study was that the cytosolic redox state of cell mutants lacking functional peroxisomes was more reductive than that of control cells.

Hyper-Hyper is a genetically encoded fluorescent biosensor that has a high sensitivity and specificity for $\mathrm{H}_{2} \mathrm{O}_{2}$ (Belousov et al. 2006). The probe consists of the regulatory domain of the Escherichia coli transcription factor OxyR (OxyR-RD), a positive regulator of $\mathrm{H}_{2} \mathrm{O}_{2}$-inducible genes, that is inserted into a circularly permuted YFP (cpYFP). Upon exposure of OxyR-RD to $\mathrm{H}_{2} \mathrm{O}_{2}$, a disulfide bond is formed between two cysteine residues, and this in turn induces a conformational change that alters cpYFP sufficiently to shift its excitation maximum from 420 to $500 \mathrm{~nm}$. As these $\mathrm{H}_{2} \mathrm{O}_{2}$ dependent changes are fully reversible, changes in the fluorescence ratios of Hyper (emission peak: $516 \mathrm{~nm}$ ) upon excitation at 420 and $500 \mathrm{~nm}$ reflect alterations in local $\mathrm{H}_{2} \mathrm{O}_{2}$-levels (Belousov et al.

2006). However, in this context, it is essential to point out that Hyper measurements are strongly influenced by $\mathrm{pH}$, and therefore it is advised to simultaneously monitor local changes in $\mathrm{pH}$ (see below). By employing targeted variants of Hyper, it has been shown that (i) in plants, peroxisomal catalase activity and $\mathrm{H}_{2} \mathrm{O}_{2}$-levels are inversely correlated (Costa et al. 2010), and (ii) in mammals, the toxicity of excess long chain nonesterified fatty acids in insulin-producing $\beta$ cells is mediated by $\mathrm{H}_{2} \mathrm{O}_{2}$ derived from peroxisomal, but not mitochondrial $\beta$-oxidation (Elsner et al. 2011).

Bodipy-PTS1 - To detect lipid peroxidation in peroxisomes in living cells, Wirtz and coworkers covalently linked a C-terminal peroxisomal matrix protein targeting signal (PTS1) to C2-Bodipy ${ }^{581 / 591}$, a membrane-permeable oxidation-sensitive fluorescent lipid peroxidation probe (Dansen and Wirtz 2001). As the red fluorescence of this fatty acid analogue (emission peak: $595 \mathrm{~nm}$ ) shifts to green fluorescence (emission peak: $520 \mathrm{~nm}$ ) upon oxidation, it can be used as a ratiometric indicator of free radical processes that have the potential 
to oxidize lipids in the peroxisomal membrane. For reasons that are not clear, this probe has - until now - only been used to show that the intraperoxisomal redox state in rat fibroblasts becomes more oxidized upon incubation of the cells with phytanic acid or arachidonic acid, which are substrates for peroxisomal $\alpha$ - and $\beta$-oxidation, respectively.

\section{Redox Modulators}

Activators and inhibitors of $\mathrm{H}_{2} \mathrm{O}_{2}$ production - Peroxisomes contain copious amounts of enzymes that can produce or degrade $\mathrm{H}_{2} \mathrm{O}_{2}$ (Antonenkov et al. 2010). Over the years, it has become clear that the expression levels and activities of these enzymes can be influenced by several factors. Examples include the induction of peroxisomal lipid metabolism in rodents fed a diet containing fibrate drugs, industrial phthalate ester plasticizers, xenobiotics, or high fat (Kozawa et al. 2011). Importantly, long-term treatment of rats with these compounds results in disproportionate increases in $\mathrm{H}_{2} \mathrm{O}_{2}$-generating enzymes and catalase, and this in turn has been linked to increased oxidative stress and DNA damage (Kasai et al. 1989). These findings have been further substantiated by other studies, which showed that overexpression of ACOX1 can activate NF- $\kappa \mathrm{B}$ DNA binding activity in a substrate concentration-dependent manner (Li et al. 2000), and the toxicity of long chain non-esterified fatty acids in insulin-producing $\beta$-cells is caused by $\mathrm{H}_{2} \mathrm{O}_{2}$ derived from peroxisomal $\beta$-oxidation (Elsner et al. 2011). Note that the results of the first study indirectly indicate that it is most likely possible to modulate the intraperoxisomal redox state by altering the activities of other bona fide $\mathrm{H}_{2} \mathrm{O}_{2}$-producing peroxisomal enzymes. One attractive candidate is D-amino acid oxidase (DAO). Indeed, a nuclear-targeted variant of this enzyme has already been successfully used to study $\mathrm{H}_{2} \mathrm{O}_{2}$-dependent signaling events in the nucleus (Halvey et al. 2007). Note that exposure of the cells to DAO substrates (e.g. D-proline and N-acetyl-D-alanine) is most likely to result in less nonperoxisomal oxidative stress than exposure of the cells to free fatty acids (Soardo et al. 2011). Another attractive approach to modulate the peroxisomal redox state involves the up- or downregulation of peroxisomal antioxidant enzyme activities. Such experiments have 
already been successfully performed for catalase. For example, it has been shown that restoration of peroxisomal catalase import (e.g. by protein transduction of catalase-SKL, a catalase analogue with enhanced peroxisome targeting efficiency) can restore a normal oxidative state in aging cells (Titorenko and Terlecky 2011).

KillerRed-PTS1 - KillerRed is a red fluorescent photosensitizer that efficiently generates ROS upon green light illumination (Bulina et al. 2006). As such, targeted variants of this protein can be used as powerful tools to study the downstream effects of local ROS production in a spatiotemporally controlled manner. We recently employed a peroxisomal variant of KillerRed to study the downstream effects of peroxisome-derived ROS (Ivashchenko et al. 2011). This study revealed that generation of excess ROS inside peroxisomes quickly perturbs the mitochondrial redox balance and leads to mitochondrial fragmentation.

\subsubsection{Ion Signaling}

As hydrogen $\left(\mathrm{H}^{+}\right)$and calcium $\left(\mathrm{Ca}^{2+}\right)$ ions are potent modulators of essentially all biological processes (Swietach et al. 2013), it is of great scientific value to gain a better understanding of how changes in peroxisomal metabolism influence subcellular homeostasis of these molecules. In the following paragraphs, we discuss the biosensors that have already been used to monitor these signaling ions in peroxisomes in living cells.

SNAFL-2-PTS1 - SNAFL-2-PTS1 is a cell-permeable fluorescent reporter molecule that can be used to monitor the intraperoxisomal $\mathrm{pH}$ in living cells (Dansen et al. 2000). It consists of a seminaphtofluorescein (SNAFL-2) moiety that is covalently linked to a PTS1 sequence. As the probe can be present in a protonated or deprotonated state (pKA 7.7), and the acidic (excitation peak: $488 \mathrm{~nm}$; emission peak: $546 \mathrm{~nm}$ ) and basic (excitation peak: $568 \mathrm{~nm}$; emission peak: $625 \mathrm{~nm}$ ) forms have different spectral characteristics, the ratio of the red and green fluorescence intensities can be used to calculate the $\mathrm{pH}$ by comparing these values with a calibration curve. By using this biosensor, these authors have demonstrated that perox- 
isomes in human fibroblasts have an internal $\mathrm{pH}$ of \pm 8.2 . However, peroxisomes in fibroblasts from patients lacking Pex $7 \mathrm{p}$, the import receptor for peroxisomal matrix proteins containing an $\mathrm{N}$-terminal peroxisomal targeting signal (PTS2), have a $\mathrm{pH}$ of \pm 6.5 (Dansen et al. 2000). The pathophysiological relevance of this observation remains to be established.

pHluorin-PTS1 - An alternative method to measure the intraperoxisomal $\mathrm{pH}$ in a non-invasive manner is by using pHluorin-PTS1, a peroxisomally located $\mathrm{pH}$-sensitive mutant of GFP (Jankowski et al. 2001). This $\mathrm{pH}$-sensor has a bimodal excitation spectrum (with peaks at $395 \mathrm{~nm}$ and $475 \mathrm{~nm}$ ) and an emission maximum at $509 \mathrm{~nm}$. As, upon acidification, the protein's excitation peak near $395 \mathrm{~nm}$ increases at the expense of the peak near $475 \mathrm{~nm}$, the corresponding emission ratio can - after calibration - provide an accurate estimate of the probe's local $\mathrm{pH}$ environment. Interestingly, by employing this biosensor, these authors found that mammalian peroxisomes do not regulate their own $\mathrm{pH}$, but that their $\mathrm{pH}$ resembles that of the cytosol (pH 6.9-7.1). Note that this finding is in conflict with that obtained by SNAFL-2-PTS1 (Dansen et al. 2000). Here it is worth mentioning that the pKa of pHluorin is 6.5 (Miesenböck et al. 1998), which is most likely too low to enable reliable measurements in case of high $\mathrm{pH}$. To bring more clarity to this issue, the use of a peroxisome-targeted variant of $\mathrm{pHRed}(\mathrm{pKa} \sim 7.8)$, a recently developed ratiometric red fluorescent $\mathrm{pH}$ sensor, may offer an alternative (Tantama et al. 2011).

$D 3 c p v-P T S 1-\mathrm{D} 3 \mathrm{cpv}$ is a computationally designed genetically encoded high-affinity FRET $\mathrm{Ca}^{2+}$ sensor that has been optimized to monitor calcium even in the presence of a large excess of native calmodulin (Palmer et al. 2006). The probe has one excitation peak near $425 \mathrm{~nm}$ and two emission maxima near 480 and $540 \mathrm{~nm}$. Upon binding to $\mathrm{Ca}^{2+}$, the protein's fluorescence emission peak near 540 $\mathrm{nm}$ is increased at the expense of the peak near $480 \mathrm{~nm}$. Therefore, the 540/480 nm ratio can be used to monitor $\mathrm{Ca}^{2+}$. By appending a PTS1 sequence to this probe, Pozzan and colleagues could demonstrate that (i) the $\mathrm{Ca}^{2+}$ concentration of peroxisomes in living mammalian cells at rest is similar to that of the cytosol, and (ii) perox- 
isomes do not act as $\mathrm{Ca}^{2+}$ stores from which $\mathrm{Ca}^{2+}$ can be mobilized upon stimulation (Drago et al. 2008).

PeroxAEQ - Aequorin is a $\mathrm{Ca}^{2+}$-sensitive photoprotein that is composed of two distinct units: the apoprotein apoaequorin, which has three high-affinity binding sites for $\mathrm{Ca}^{2+}$; and the prosthetic group coelenterazine, a blue light-emitting membrane-permeable molecule found in many aquatic organisms (Rizzuto et al. 1992). Both components reconstitute spontaneously to form a functional protein that, upon $\mathrm{Ca}^{2+}$-binding, undergoes a conformational change (Chudakov et al. 2010). This change triggers the conversion of the protein into an oxygenase, which oxidizes coelenterazine to coelenteramide. The subsequent relaxation of coelenteramide to its ground state results in the emission of blue light (near $469 \mathrm{~nm}$ ), which can be detected by a luminometer. Palmieri and colleagues generated a PTS1-tagged variant of apoaequorin, termed PeroxAEQ, to study the dynamics of free $\mathrm{Ca}^{2+}$ in the peroxisomal matrix (Lasorsa et al. 2008). In contrast to the findings reported by Pozzan and colleagues (see above), these researchers found that (i) the $\mathrm{Ca}^{2+}$ concentration of peroxisomes in living mammalian cells at rest is approximately 20 times higher than that of the cytosol, and (ii) peroxisomes transiently take up calcium upon stimulation with agonists that induce $\mathrm{Ca}^{2+}$ release from intracellular stores. Further work needs to be done to reconcile these apparently conflicting studies.

\section{Conclusions and Perspectives}

Hidden in the shadow of mitochondria, peroxisomes have only recently begun to emerge as potentially important signaling platforms in various physiological and pathological processes (Titorenko and Terlecky 2011). As illustrated in Section 2, an increasing amount of evidence supports the idea that these organelles are at the crossroads between lipid metabolism, redox signaling, and inflammation. This is further emphasized by the observation that dysfunctional peroxisomes sensitize cells and organs for oxidative stress, a condition associated with the initiation and progression of age-related disorders such as type 2 diabetes, cancer, and neurodegenerative diseases 
(Fransen et al. 2013). In this context, deciphering the role of peroxisomes in various cellular signaling processes is of paramount importance, both for basic and translational research. Over the past years, through the development of novel and improved methods and technologies, this research field has fundamentally changed. In this chapter, we have mainly focused on the use of a wide range of fluorescent probes that are suitable for live cell imaging (see Section 3). However, as (i) each probe has its intrinsic limitations (e.g. Hyper measurements are strongly influenced by $\mathrm{pH}$ ), and (ii) different probes sometimes yield different results (e.g. see Sect. 3.2: compare SNAFL-2-PTS1 with pHluorin-PTS1, and D3cpv-PTS1 with PeroxAEQ), results obtained from live cell microscopy should be confirmed by independent methods (e.g. biochemical assays, redox proteomics, microarray analysis, etc.) to avoid misinterpretation. Finally, it is needless to say that we are still far from understanding the mechanistic details of how peroxisomes are embedded in cellular signaling networks. For example, the proximal targets of peroxisomal ROS are largely unknown; the molecular mechanisms underlying stress-related communication events between peroxisomes and mitochondria remain to be discovered; and it is even not yet clear under which conditions peroxisomes act as net sources or sinks of ROS. Finding answers to these and many other questions will certainly advance our understanding of how peroxisomes are integrated into developmental programs, and how dysregulation of peroxisome homeostasis may lead to pathological conditions.

Acknowledgments MF is supported by grants from the "Fonds voor Wetenschappelijk Onderzoek- Vlaanderen (Onderzoeksproject G.0754.09)" and the "Bijzonder Onderzoeksfonds van de KU Leuven (OT/09/045)." MN is supported by a FLOF fellowship from the Department of Cellular and Molecular Medicine, KU Leuven. BW is a recipient of a DBOF fellowship (DBOF/10/059) from the KU Leuven.

\section{References $^{1}$}

Antonenkov VD, Grunau S, Ohlmeier S et al (2010) Peroxisomes are oxidative organelles. Antioxid Redox Signal 13:525-537

\footnotetext{
${ }^{1}$ We apologize to colleagues whose work could not be cited due to space limitations.
} 
Baarine M, Andréoletti P, Athias A et al (2012) Evidence of oxidative stress in very long chain fatty acid-treated oligodendrocytes and potentialization of ROS production using RNA interferencedirected knockdown of ABCD1 and ACOX1 peroxisomal proteins. Neuroscience 213:1-18

Barford D (2004) The role of cysteine residues as redox-sensitive regulatory switches. Curr Opin Struct Biol 14:679-686

Beach A, Burstein MT, Richard VR et al (2012) Integration of peroxisomes into an endomembrane system that governs cellular aging. Front Physiol 3:283. doi:10.3389/fphys.2012.00283

Belousov VV, Fradkov AF, Lukyanov KA et al (2006) Genetically encoded fluorescent indicator for intracellular hydrogen peroxide. Nat Methods 3:281-286

Bikman BT, Summers SA (2011) Ceramides as modulators of cellular and whole-body metabolism. J Clin Invest 121:4222-4230

Bottelbergs A, Verheijden S, Van Veldhoven PP et al (2012) Peroxisome deficiency but not the defect in ether lipid synthesis causes activation of the innate immune system and axonal loss in the central nervous system. J Neuroinflammation 9:61. doi:10.1186/1742-2094-9-61

Boveris A, Oshino N, Chance B (1972) The cellular production of hydrogen peroxide. Biochem J 128:617-630

Braverman NE, Moser AB (2012) Functions of plasmalogen lipids in health and disease. Biochim Biophys Acta 1822:1442-1452

Brodde A, Teigler A, Brugger B et al (2012) Impaired neurotransmission in ether lipid-deficient nerve terminals. Hum Mol Genet 21:2713-2724

Bulina ME, Chudakov DM, Britanova OV et al (2006) A genetically encoded photosensitizer. Nat Biotechnol 24:95-99

Chen H, Jiang Z (2013) The essential adaptors of innate immune signaling. Protein Cell 4:27-39

Chen X, Liang H, Van Remmen H et al (2004) Catalase transgenic mice: characterization and sensitivity to oxidative stress. Arch Biochem Biophys 422:197-210

Chen ZH, Niki E (2006) 4-hydroxynonenal (4-HNE) has been widely accepted as an inducer of oxidative stress. Is this the whole truth about it or can 4-HNE also exert protective effects? IUBMB Life 58:372-373 
Chudakov DM, Matz MV, Lukyanov S et al (2010) Fluorescent proteins and their applications in imaging living cells and tissues. Physiol Rev 90:1103-1163

Costa A, Drago I, Behera S et al (2010) $\mathrm{H}_{2} \mathrm{O}_{2}$ in plant peroxisomes: an in vivo analysis uncovers a $\mathrm{Ca}^{2+}$-dependent scavenging system. Plant J 62:760-772

Dansen TB, Wirtz KW (2001) The peroxisome in oxidative stress. IUBMB Life 51:223-230

Dansen TB, Wirtz KW, Wanders RJ et al (2000) Peroxisomes in human fibroblasts have a basic $\mathrm{pH}$. Nat Cell Biol 2:51-53

Del Río LA (2011) Peroxisomes as a cellular source of reactive nitrogen species signal molecules. Arch Biochem Biophys 506:1-11

Delille HK, Agricola B, Guimaraes SC et al (2010) Pex11pbetamediated growth and division of mammalian peroxisomes follows a maturation pathway. J Cell Sci 123:2750-2762

Diano S, Liu ZW, Jeong JK et al (2011) Peroxisome proliferationassociated control of reactive oxygen species sets melanocortin tone and feeding in diet-induced obesity. Nat Med 17:1121-1127

Dikalov S, Griendling KK, Harrison DG (2007) Measurement of reactive oxygen species in cardiovascular studies. Hypertension 49:717-727

Dixit E, Boulant S, Zhang Y et al (2010) Peroxisomes are signaling platforms for antiviral innate immunity. Cell 141:668-681

Doulias PT, Tenopoulou M, Greene JL et al (2013) Nitric oxide regulates mitochondrial fatty acid metabolism through reversible protein S-nitrosylation. Sci Signal 6 (256):rs1. doi:10.1126/scisignal.2003252

Drago I, Giacomello M, Pizzo P et al (2008) Calcium dynamics in the peroxisomal lumen of living cells. J Biol Chem 283:1438414390

El Hajj HI, Vluggens A, Andreoletti P et al (2012) The inflammatory response in acyl-CoA oxidase 1 deficiency (pseudoneonatal adrenoleukodystrophy). Endocrinology 153:2568-2575

Elsner M, Gehrmann W, Lenzen S (2011) Peroxisome-generated hydrogen peroxide as important mediator of lipotoxicity in insulin-producing cells. Diabetes 60:200-208

Fourcade S, López-Erauskin J, Galino J et al (2008) Early oxidative damage underlying neurodegeneration in Xadrenoleukodystrophy. Hum Mol Genet 17:1762-1773 
Fransen M (2012) Peroxisome dynamics: molecular players, mechanisms, and (dys)functions. ISRN Cell Biology 2012: article ID 714192. doi:10.5402/2012/714192

Fransen M, Nordgren M, Wang B et al (2012) Role of peroxisomes in ROS/RNS-metabolism: implications for human disease.

Biochim Biophys Acta 1822:1363-1373

Fransen M, Nordgren M, Wang B et al (2013) Aging, age-related diseases and peroxisomes. Subcell Biochem 69:45-65

Gutscher M, Sobotta MC, Wabnitz GH et al. (2009) Proximitybased protein thiol oxidation by $\mathrm{H}_{2} \mathrm{O}_{2}$-scavenging peroxidases. $\mathbf{J}$ Biol Chem 284:31532-31540.

Halvey PJ, Hansen JM, Johnson JM et al (2007) Selective oxidative stress in cell nuclei by nuclear-targeted D-amino acid oxidase. Antioxid Redox Signal 9:807-816

Hanson GT, Aggeler R, Oglesbee D et al (2004) Investigating mitochondrial redox potential with redox-sensitive green fluorescent protein indicators. J Biol Chem 279:13044-13053

Hasegawa K, Wakino S, Yoshioka K et al (2010) Kidney-specific overexpression of Sirt1 protects against acute kidney injury by retaining peroxisome function. J Biol Chem 285:13045-13056

Hori Y, Kikuchi K (2013) Protein labeling with fluorogenic probes for no-wash live-cell imaging of proteins. Curr Opin Chem Biol doi:10.1016/j.cbpa.2013.05.015

Huybrechts SJ, Van Veldhoven PP, Brees C et al (2009) Peroxisome dynamics in cultured mammalian cells. Traffic 10:1722-1733

Hwang I, Lee J, Huh JY et al (2012) Catalase deficiency accelerates diabetic renal injury through peroxisomal dysfunction. Diabetes 61:728-738

Ivashchenko O, Van Veldhoven PP, Brees C et al (2011) Intraperoxisomal redox balance in mammalian cells: oxidative stress and interorganellar cross-talk. Mol Biol Cell 22:1440-1451

Jankowski A, Kim JH, Collins RF et al (2001) In situ measurements of the $\mathrm{pH}$ of mammalian peroxisomes using the fluorescent protein pHluorin. J Biol Chem 276:48748-48753

Karlsson M, Kurz T, Brunk UT et al (2010) What does the commonly used DCF test for oxidative stress really show? Biochem J 428:183-190 
Kasai H, Okada Y, Nishimura S et al (1989) Formation of 8hydroxydeoxyguanosine in liver DNA of rats following long-term exposure to a peroxisome proliferator. Cancer Res 49:2603-2605

Kassmann CM, Lappe-Siefke C, Baes M et al (2007) Axonal loss and neuroinflammation caused by peroxisome-deficient oligodendrocytes. Nat Genet 39:969-976

Kim PK, Mullen RT, Schumann U et al (2006) The origin and maintenance of mammalian peroxisomes involves a de novo PEX16dependent pathway from the ER. J Cell Biol 173:521-532

Kozawa S, Honda A, Kajiwara N et al (2011) Induction of peroxisomal lipid metabolism in mice fed a high-fat diet. Mol Med Rep 4:1157-1162

Lasorsa FM, Pinton P, Palmieri L et al (2008) Peroxisomes as novel players in cell calcium homeostasis. J Biol Chem 283:1530015308

Lazarow PB (2011) Viruses exploiting peroxisomes. Curr Opin Microbiol 14:458-469

Li Y, Chen R, Zhou Q et al (2012) LSm14A is a processing bodyassociated sensor of viral nucleic acids that initiates cellular antiviral response in the early phase of viral infection. Proc Natl Acad Sci USA 109:11770-11775

Li Y, Tharappel JC, Cooper S et al (2000) Expression of the hydrogen peroxide-generating enzyme fatty acyl CoA oxidase activates NF-kappaB. DNA Cell Biol 19:113-120

Los GV, Encell LP, McDougall MG et al (2008) HaloTag: a novel protein labeling technology for cell imaging and protein analysis. ACS Chem Biol 3:373-382

Lukyanov KA, Belousov VV (2013) Genetically encoded fluorescent redox sensors. Biochim Biophys Acta. doi:10.1016/j.bbagen.2013.05.030

Mast FD, Li J, Virk MK et al (2011) A Drosophila model for the Zellweger spectrum of peroxisome biogenesis disorders. Dis Model Mech 4:659-672

Miesenböck G, De Angelis DA, Rothman JE (1998) Visualizing secretion and synaptic transmission with $\mathrm{pH}$-sensitive green fluorescent proteins. Nature 394:192-195

Müller CC, Nguyen TH, Ahlemeyer B et al (2011) PEX13 deficiency in mouse brain as a model of Zellweger syndrome: abnor- 
mal cerebellum formation, reactive gliosis and oxidative stress.

Dis Model Mech 4:104-119

Palmer AE, Giacomello M, Kortemme T et al (2006) $\mathrm{Ca}^{2+}$ indicators based on computationally redesigned calmodulin-peptide pairs.

Chem Biol 13:521-530

Pettus BJ, Baes M, Busman M et al (2004) Mass spectrometric analysis of ceramide perturbations in brain and fibroblasts of mice and human patients with peroxisomal disorders. Rapid Commun Mass Spectrom 18:1569-1574

Rhodin, J (1954) Correlation of ultrastructural organization and function in normal and experimentally changed proximal tubule cells of the mouse kidney. Dissertation, Karolinska Institutet

Ribeiro D, Castro I, Fahimi HD et al (2012) Peroxisome morphology in pathology. Histol Histopathol 27:661-676

Rizzuto R, Simpson AW, Brini M et al (1992) Rapid changes of mitochondrial $\mathrm{Ca}^{2+}$ revealed by specifically targeted recombinant aequorin. Nature 358:325-327

Schwarzländer M, Fricker MD, Müller C et al (2008) Confocal imaging of glutathione redox potential in living plant cells. J Microsc 231:299-316

Singh J, Khan M, Singh I (2009) Silencing of Abcd1 and Abcd2 genes sensitizes astrocytes for inflammation: implication for Xadrenoleukodystrophy. J Lipid Res 50:135-147

Soardo G, Donnini D, Domenis L et al (2011) Oxidative stress is activated by free fatty acids in cultured human hepatocytes. Metab Syndr Relat Disord 9:397-401

Stolz DB, Zamora R, Vodovotz Y et al (2002) Peroxisomal localization of inducible nitric oxide synthase in hepatocytes. Hepatology 36:81-93

Swietach P, Youm JB, Saegusa N et al (2013) Coupled $\mathrm{Ca}^{2+} / \mathrm{H}^{+}$ transport by cytoplasmic buffers regulates local $\mathrm{Ca}^{2+}$ and $\mathrm{H}^{+}$ion signaling. Proc Natl Acad Sci USA 110:E2064-E2073

Tantama M, Hung YP, Yellen G (2011) Imaging intracellular pH in live cells with a genetically encoded red fluorescent protein sensor. J Am Chem Soc 133:10034-10037

Titorenko VI, Terlecky SR (2011) Peroxisome metabolism and cellular aging. Traffic 12:252-259 
Van Veldhoven PP (2010) Biochemistry and genetics of inherited disorders of peroxisomal fatty acid metabolism. J Lipid Res 51:2863-2895

Vasko R, Ratliff BB, Bohr S et al (2013) Endothelial peroxisomal dysfunction and impaired pexophagy promotes oxidative damage in lipopolysaccharide-induced acute kidney injury. Antioxid Redox Signal. doi:10.1089/ars.2012.4768

Verheijden S, Bottelbergs A, Krysko O et al (2013) Peroxisomal multifunctional protein-2 deficiency causes neuroinflammation and degeneration of Purkinje cells independent of very long chain fatty acid accumulation. Neurobiol Dis (in press). doi:10.1016/j.nbd.2013.06.006

Walton PA, Pizzitelli M (2012) Effects of peroxisomal catalase inhibition on mitochondrial function. Front Physiol 3:108. doi:10.3389/fphys.2012.00108

Wang S, Li N, Pan W et al (2012) Advances in functional fluorescent and luminescent probes for imaging intracellular smallmolecule reactive species. Trend Anal Chem 39:3-37

Yang Y, Song Y, Loscalzo J (2007) Regulation of the protein disulfide proteome by mitochondria in mammalian cells. Proc Natl Acad Sci USA 104:10813-10817

Yano T, Oku M, Akeyama N et al (2010) A novel fluorescent sensor protein for visualization of redox states in the cytoplasm and in peroxisomes. Mol Cell Biol 30:3758-3766 


\section{Figure Legend}

Fig. 1. Model depicting the role of peroxisomes as intracellular signaling platforms. Peroxisomes play a central role in cellular lipid and ROS metabolism, and substantial evidence supports the view that the functional state of these organelles can influence redox-, lipid-, and inflammatory-mediated signaling pathways. Peroxisomes can also activate and propagate antiviral innate immune responses via peroxisomal MAVS, an adaptor protein for pattern recognition receptors such as RIG-I-like receptor (RLR) and LSm14A. Taken together, alterations in peroxisome activity may - directly or indirectly - modulate mitochondrial function and trigger changes in nuclear gene expression (see text for more details). This in turn may regulate cell fate by activating cytoprotective or cytotoxic signaling responses. To visualize peroxisomes in living cells, one can employ fluorescence microscopy in combination with selective fluorescent labeling techniques. These include genetically encoded (photoactivatable (PA)) fluorescent proteins (FPs), fluorescent timers, and self-labeling proteins (e.g. HaloTag), all of which can be tagged with a peroxisomal targeting signal (PTS). To monitor local changes in peroxisomal redox and ion homeostasis, one can use organelle-targeted biosensors that are capable of detecting nonspecific ROS, GSH/GSSG, $\mathrm{H}_{2} \mathrm{O}_{2}$, lipid peroxides (LPOs), $\mathrm{H}^{+}$, or $\mathrm{Ca}^{2+}$. To study how peroxisomal metabolism affects the global cellular redox state, one can also use non-targetable redox sensors, such as $\mathrm{H}_{2}$ DCFDA, dihydroethidine, and C11-Bodipy ${ }^{581 / 591}$. Finally, to modulate the peroxisomal redox state, one can alter the expression levels and/or activity of peroxisomal ROSmodulating enzymes or employ peroxisomal KillerRed (see text for more details). The organelles are not drawn to scale. 3-AT, 3-amino-1,2,4-triazole; ACOX1, acyl-CoA oxidase 1; DAO, Damino acid oxidase; MT, mitochondrion; NU, nucleus; PO, peroxisome. 


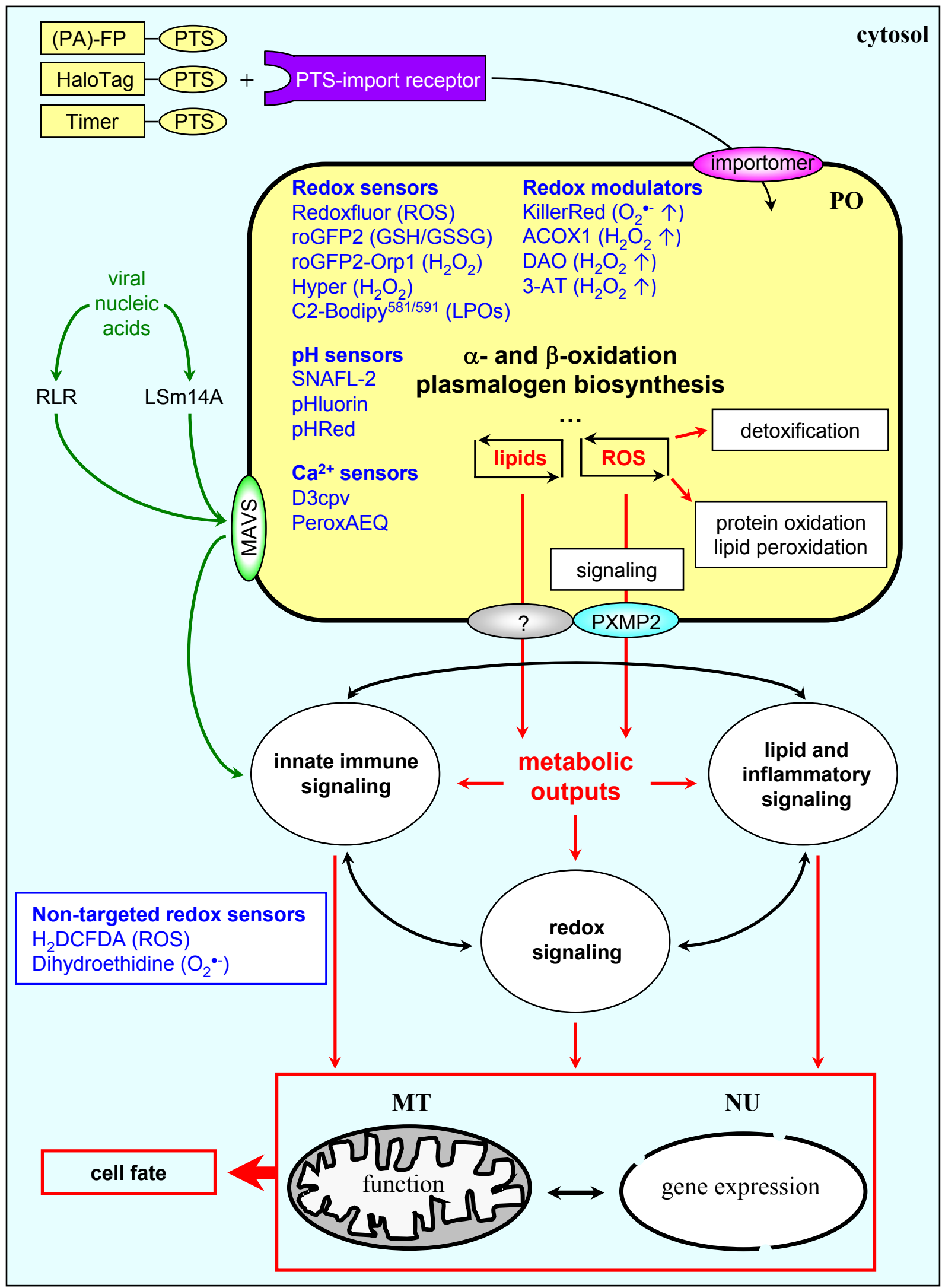

